\title{
Synthesis and Biological Activities of Novel Arylazopyrazolones Substituted with Thiazolyhydrazone
}

\author{
Purvesh J. Shah* \\ Department of Chemistry, Shree P. M. Patel Institute of P. G. Studies \& Research in Science, \\ Sardar Patel University, Vallabh Vidyanagar, Anand 388120, Gujarat, India. \\ *E-mail: purvesh23184@gmail.com
}

(Received October 13, 2013; Accepted December 17, 2013)

\begin{abstract}
The 4-(1H)-benzotriazoyl methyl amino benzoate 3 was prepared by Mannich reaction of benzotriazole 1, ethyl-paminobenzoate 2 and formaldehyde. The prepared compound 3 then react with hydrazine hydrate results in the 4- $(1 \mathrm{H})$-benzotriazoyl methyl amino benzoyl hydrazide 4. This compound on condensation with pre-prepared different ethyl 2-(2-(4-(4-substituted phenyl)thiazol-2-yl)hydrazono)-3-oxobutanoates 6a-d, furnished 1-(4-((1H-benzo[d] [1,2,3] triazol-1-yl)methyl amino) benzoyl)-4-(2-(4-(4-substituted phenyl)thiazol-2-yl) hydrazono)-3-methyl-1H-pyrazol-5(4H)-one 7a-d. All the compounds 7a-d was characterized by spectral studies. The compounds showed significant antimicrobial activity against various bacteria and fungi.
\end{abstract}

Key words: Pyrazole, Benzotriazole, Mannich reaction, Spectral studies, Antimicrobial activity

\section{INTRODUCTION}

Many pyrazolines and substituted pyrazolines derivatives are well known for their biological and pharmacological activities, ${ }^{1-5}$ which exhibit an anti-inflammatory, ${ }^{6}$ fungicidal, ${ }^{7,8}$ bactericidal, antipyretic, ${ }^{9,10}$ anti depressant, ${ }^{11-14}$ anticonvulsant $^{13,14}$ and protein kinase inhibitors. ${ }^{15,16}$ These pyrazolone derivatives were investigated as thermal stabilizers for rigid PVC. ${ }^{17,18}$ On the other hand, many azopyrazolone dyes have been utilized as chromogenic reagents forcolourimetric determinations ${ }^{19,20}$ and as indicator for complexometric titrations. ${ }^{21}$ Also, there are some arylazopyrazolone dyes having potent antimicrobial activities. $^{22}$ Arylazopyrazoles are generally prepared by combination of aryl-azo-ethyl actoacetate derivatives and hydrazine derivatives. $^{23-27}$ The benzotriazole is found as an important heterocyclic compound. They reveal valuable pharmacological properties and clinical applications. ${ }^{28-31}$ It is prime application is as corrosion inhibitors for copper or copper alloys. ${ }^{32}$ The area in which the merged molecule like aryl azo pyrazole-benzotriazole has not been developed in spite of good biological properties of both these compounds. Hence the present paper comprises the synthesis and characterization of aryl azo pyrazole-benzotriazole derivatives shown in Scheme 1.

\section{EXPERIMENTAL}

All chemicals used were of laboratory grade. Ethyl-4- amino benzoate, Benzotriazole and various substituted 2amino-4-phenyl thiazole derivatives 5a-d were prepared by reported method. ${ }^{33,34-36}$ Melting points were determined in open capillary tubes and were uncorrected. IR spectra were recorded in $\mathrm{KBr}$ pellets on a Nicolet 760D spectrometer. ${ }^{1} \mathrm{H} \mathrm{NMR}$ and ${ }^{13} \mathrm{C} \mathrm{NMR}$ spectra were recorded in DMSO with TMS as internal standard on a Bruker spectrometer at $400 \mathrm{MHz}$ and $100 \mathrm{MHz}$, respectively. LCMS of selected samples taken on LC-MSD-Trap-SL_01046 instrument.

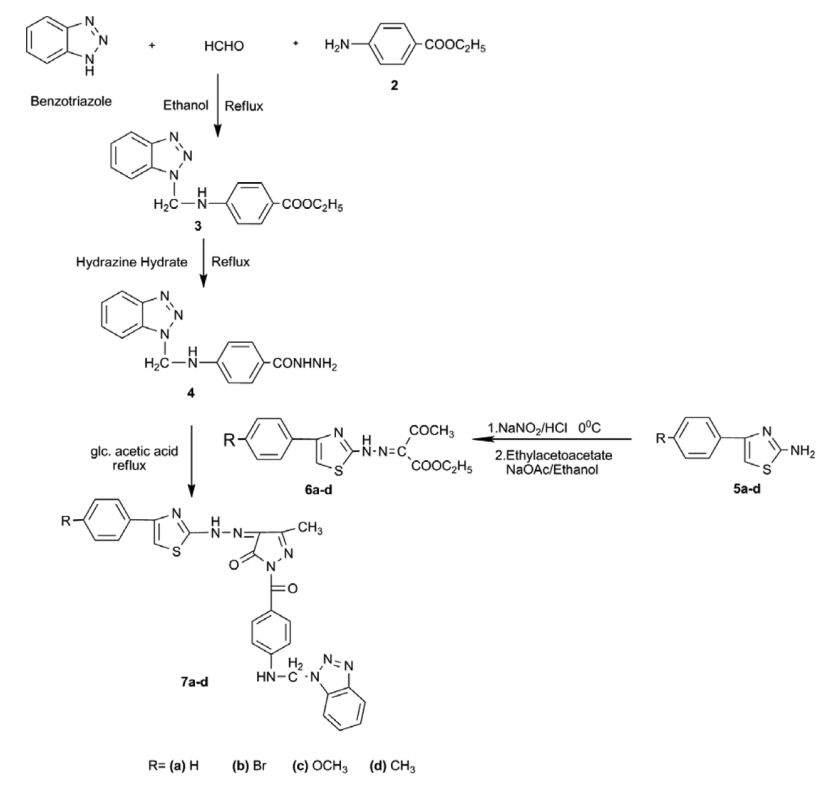

Scheme 1. Synthesis of arylazopyrazole derivatives. 
Synthesis of 4-(1H)-benzotriazolyl methyl aminobenzoate 3

A mixture of $1 \mathrm{H}$-Benzotriazole 1 ( 0.02 mole), formaldehyde ( 0.02 mole) and ethyl-4-amino benzoate 2 ( 0.02 mole $)$ in ethanol $(50 \mathrm{ml})$ was heated under reflux for $4 \mathrm{~h}$. Subsequequently, ethanol was distilled off and the pasty mass obtained, which was triturated with petroleum ether (40$\left.60{ }^{\circ} \mathrm{C}\right)$. The solid 4-(1H)-benzotriazolyl methylamino benzoate 3, which was isolated and dried. Yield 68\%, m.p. $146-147^{\circ} \mathrm{C}$. IR ( $\left.v, \mathrm{~cm}^{-1}, \mathrm{KBr}\right) 3034-3086$ (C-H aromatic), $2965\left(\mathrm{CH}_{2}\right), 1197-1255(\mathrm{C}-\mathrm{N}), 725(\mathrm{C}=\mathrm{O}$ of ester), 28902910, 1456 (C-H). ${ }^{1} \mathrm{H}$ NMR (400 MHz, $\delta$, ppm, DMSOd $\mathrm{d}_{6} 5.7\left(\mathrm{~s}, 2 \mathrm{H}, \mathrm{CH}_{2}\right), 3.2(\mathrm{~s}, 1 \mathrm{H}, \mathrm{NH}), 6.56-8.2(\mathrm{~m}, 8 \mathrm{H}, \mathrm{Ar}-\mathrm{H})$, 4.32 (q, $\left.2 \mathrm{H},-\mathrm{O}-\mathrm{CH}_{2}\right), 1.32\left(\mathrm{t}, 3 \mathrm{H}, \mathrm{CH}_{3}\right) .{ }^{13} \mathrm{C} \mathrm{NMR}(100 \mathrm{MHz}$, $\delta$, ppm, DMSO) 114.3-149.1 ( $\mathrm{Ar}-\mathrm{C}), 75.7\left(\mathrm{CH}_{2}\right), 13.9\left(\mathrm{CH}_{3}\right)$, $62.1\left(\mathrm{CH}_{2}\right), 170.4(\mathrm{CO})$. LC-MS: $m / z 305\left(\mathrm{M}^{+}\right)$. Anal. Calcd for $\mathrm{C}_{16} \mathrm{H}_{16} \mathrm{~N}_{4} \mathrm{O}_{2}(296) \mathrm{C}, 64.85 ; \mathrm{H}, 5.44 ; \mathrm{N}, 18.91$. Found: C, 64.8; H, 5.4; N, 18.9.

Synthesis of 4-(1H)-benzotriazolyl methyl amino benzoyl hydrazide 4

4-(1H)-benzotriazolylmethyl amino benzoate 3 ( 0.05 mole) was refluxed with hydrazine hydrate $(0.05$ mole $)$ in absolute ethanol for 8 to 10 hours. It was cooled and kept overnight. The solid so obtained was filtered and recrystallized from ethanol. Yield $63 \%$, m.p. $77-78^{\circ} \mathrm{C}$. IR $\left(v, \mathrm{~cm}^{-1}, \mathrm{KBr}\right)$ 3034-3086 (C-H aromatic), $2965\left(\mathrm{CH}_{2}\right), 1197-1255(\mathrm{C}-\mathrm{N})$, $1725\left(\mathrm{C}=\mathrm{O}\right.$ of ester), 3450, $1630\left(\mathrm{NH}_{2}\right) .{ }^{1} \mathrm{H}$ NMR (400 $\left.\mathrm{MHz}, \delta, \mathrm{ppm}, \mathrm{DMSO}-\mathrm{d}_{6}\right) 3.95\left(\mathrm{~s}, 2 \mathrm{H}, \mathrm{NH}_{2}\right), 8.21-6.56$ (m, 8H, Ar-H), $9.66(\mathrm{~s}, 1 \mathrm{H}, \mathrm{CONH}), 5.7$ (s, 2H, $\left.\mathrm{CH}_{2}\right), 3.2$ (s, 1H, NH). ${ }^{13} \mathrm{C} \mathrm{NMR}(100 \mathrm{MHz}, \delta, \mathrm{ppm}, \mathrm{DMSO}) 114.3-$ 149.1 (Ar-C), $75.7\left(\mathrm{CH}_{2}\right), 170.4$ (CO). LC-MS: m/z 291 $\left(\mathrm{M}^{+}\right)$. Anal. Calcd for $\mathrm{C}_{14} \mathrm{H}_{14} \mathrm{~N}_{6} \mathrm{O}$ (282): Calcd.: C, 59.56; H, 5.00; N, 29.77. Found: C, 59.5; H, 4.9; N, 29.7.

General Procedure for the Synthesis of Various Ethyl 3-oxo-2-(2-(substituted phenyl thiazol-2-yl) hydrazono) butanoate $6 \mathbf{a}-\mathbf{d}$

To a various substituted phenyl thiazole Amine 5a-d $(0.01 \mathrm{~mole})$ was dissolved in a mixture of $\mathrm{HCl}(8 \mathrm{ml})$ and water $(6 \mathrm{ml})$ and cooled to $0{ }^{\circ} \mathrm{C}$ in ice bath. To it a cold aqueous solution of sodium nitrate ( 0.03 mole) was added. The diazonium salt solution was filtered into a cooled solution of ethyl actoacetate $(0.01$ mole $)$ and sodium acetate $(0.12 \mathrm{~mole})$ in ethanol $(50 \mathrm{ml})$. The resulting solid was washed with water and recrystallized from $\mathrm{EtOH} / \mathrm{MeOH}$.

\section{Physical Properties and Spectral Data}

Ethyl 2-(2-(4-(p-phenyl) thiazol-2-yl) hydrazono)-3 oxobutanoate 6a
Yield 78\%; m.p. $145-147{ }^{\circ} \mathrm{C}$. IR ( $\left(v, \mathrm{~cm}^{-1}, \mathrm{KBr}\right) 3030-$ 3085 (C-H aromatic), 2920, $1465\left(\mathrm{CH}_{3}, \mathrm{CH}_{2}\right), 1290(\mathrm{C}-\mathrm{N})$, 1725-1765 (C=O), $1148(\mathrm{C}-\mathrm{O}), 3369(\mathrm{~N}-\mathrm{H}), 1580(\mathrm{C}=\mathrm{N})$, 1597, 1504 (Thiazole ring), $1550(\mathrm{C}-\mathrm{S}-\mathrm{C}) .{ }^{1} \mathrm{HNMR}[400$ $\mathrm{MHz}, \delta$, ppm, DMSO-d $)^{2} 1.34$ (t, 3H, $\left.\mathrm{CH}_{3}\right), 4.29$ (q, 2H, $\left.\mathrm{COCH}_{2}\right), 2.35$ (s, 3H, $\left.\mathrm{COCH}_{3}\right), 7.48-7.94$ (m, 6H, ArH),

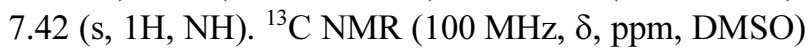
107.8-172.6 (Ar-C), 134.6 (C=N), 14.4, $27.3\left(\mathrm{CH}_{3}\right), 61.4$ $\left(\mathrm{CH}_{2}\right)$, 165.4, 195.4 (CO). LC-MS: $m / z 329\left(\mathrm{M}^{+}\right)$. Anal. Calcd for $\mathrm{C}_{15} \mathrm{H}_{15} \mathrm{~N}_{3} \mathrm{O}_{3} \mathrm{~S}$ (317): C, 56.77; $\mathrm{H}, 4.76 ; \mathrm{N}, 13.24$; S, 10.10. Found: C, 56.7; H, 4.7; N, 13.2; S, 10.0 .

Ethyl 2-(2-(4-(4-bromophenyl)thiazol-2-yl) hydrazono)3-oxobutanoate 6 b

Yield 74\%; m.p. $142-143{ }^{\circ} \mathrm{C}$. IR $\left(v, \mathrm{~cm}^{-1}, \mathrm{KBr}\right) 3030-$ 3085 (C-H aromatic), 2920, $1465\left(\mathrm{CH}_{3}, \mathrm{CH}_{2}\right), 1290(\mathrm{C}-\mathrm{N})$, 1725-1765 (C=O), $1148(\mathrm{C}-\mathrm{O}), 3369(\mathrm{~N}-\mathrm{H}), 1580(\mathrm{C}=\mathrm{N})$, 1597, 1504 (Thiazole ring), $1550(\mathrm{C}-\mathrm{S}-\mathrm{C}), 550(\mathrm{C}-\mathrm{Br})$. ${ }^{1} \mathrm{H} \mathrm{NMR}\left(400 \mathrm{MHz}, \delta\right.$, ppm, DMSO-d $\left.\mathrm{d}_{6}\right) 1.34\left(\mathrm{t}, 3 \mathrm{H}, \mathrm{CH}_{3}\right.$ ), 4.29 (q, $2 \mathrm{H}, \mathrm{COCH}_{2}$ ), 2.35 (s, 3H, $\left.\mathrm{COCH}_{3}\right), 7.42-7.90$ (m, 5H, ArH), 7.42 (s, 1H, NH). ${ }^{13} \mathrm{C}$ NMR (100 MHz, $\delta$, ppm, DMSO) 114.8-143.6 (Ar-C), 123.8 (C-Br), 14.2, $26.9\left(\mathrm{CH}_{3}\right), 61.4\left(\mathrm{CH}_{2}\right), 165.4,195.4(\mathrm{CO})$. LC-MS: $\mathrm{m} / \mathrm{z}$ $405\left(\mathrm{M}^{+}\right)$. Anal. Calcd for $\mathrm{C}_{15} \mathrm{H}_{14} \mathrm{~N}_{3} \mathrm{O}_{3} \mathrm{BrS}$ (394): $\mathrm{C}$, 45.47; H, 3.56; N, 10.60; Br, 20.16; S, 8.09. Found C, 45.4; H, 3.5; N, 10.5; Br, 20.1; S, 8.0.

Ethyl-2-(2-(4-(4-methoxyphenyl)thiazol-2-yl)hydrazono)-3-oxobutanoate $6 \mathrm{c}$

Yield 75\%; m.p. $141-143{ }^{\circ} \mathrm{C}$. IR $\left(v, \mathrm{~cm}^{-1}, \mathrm{KBr}\right) 3030-$ 3085 (C-H aromatic), 2920, $1465\left(\mathrm{CH}_{3}, \mathrm{CH}_{2}\right), 1290$ (C$\mathrm{N})$, 1725-1765 $(\mathrm{C}=\mathrm{O}), 1148(\mathrm{C}-\mathrm{O}), 3369(\mathrm{~N}-\mathrm{H}), 1580$ $(\mathrm{C}=\mathrm{N}), 1597,1504$ (Thiazole ring), $1550(\mathrm{C}-\mathrm{S}-\mathrm{C}), 1205$ (R-O-Ar). ${ }^{1} \mathrm{H}$ NMR (400 MHz, $\delta$, ppm, DMSO-d 6 ]: 3.65 (s, 3H, $\left.\mathrm{OCH}_{3}\right), 1.34$ (t, 3H, $\left.\mathrm{CH}_{3}\right), 4.29$ (q, $2 \mathrm{H}, \mathrm{COCH}_{2}$ ), $2.35\left(\mathrm{~s}, 3 \mathrm{H}, \mathrm{COCH}_{3}\right), 7.42-7.90(\mathrm{~m}, 5 \mathrm{H}, \mathrm{ArH}), 7.42(\mathrm{~s}$, $1 \mathrm{H}, \mathrm{NH}) .{ }^{13} \mathrm{C}$ NMR $(100 \mathrm{MHz}, \delta$, ppm, DMSO) $114.8-$ $160.6(\mathrm{Ar}-\mathrm{C}), 56.2\left(\mathrm{O}-\mathrm{CH}_{3}\right), 14.2,26.9\left(\mathrm{CH}_{3}\right), 61.4\left(\mathrm{CH}_{2}\right)$, 165.4, 195.4 (CO). LC-MS: m/z $356\left(\mathrm{M}^{+}\right)$. Anal. Calcd for $\mathrm{C}_{16} \mathrm{H}_{17} \mathrm{~N}_{3} \mathrm{O}_{4} \mathrm{~S}$ (347): C, 55.32; H, 4.93; N, 12.10; S, 9.23. Found: C, 55.2; H, 4.9; N, 12.0; S, 9.0.

Ethyl 3-oxo-2-(2-(4-p-tolylthiazol-2-yl) hydrazono) butanoate $6 \mathrm{~d}$

Yield 78\%; m.p. $147-149^{\circ} \mathrm{C}$. IR $\left(v, \mathrm{~cm}^{-1}, \mathrm{KBr}\right) 3030-$ 3085 (C-H aromatic), 2920, $\left.1465 \mathrm{CH}_{3}, \mathrm{CH}_{2}\right), 1290(\mathrm{C}-\mathrm{N})$, 1725-1765 (C=O), $1148(\mathrm{C}-\mathrm{O}), 3369(\mathrm{~N}-\mathrm{H}), 1580(\mathrm{C}=\mathrm{N})$, 1597, 1504 (Thiazole ring), $1550(\mathrm{C}-\mathrm{S}-\mathrm{C}) .{ }^{1} \mathrm{H}$ NMR (400 MHz, $\delta$, ppm, DMSO-d 6 ]: 2.42 (s, 3H, $\left.\mathrm{CH}_{3}\right), 1.34$ (t, 3H, 
$\left.\mathrm{CH}_{3}\right), 4.29$ (q, 2H, $\left.\mathrm{COCH}_{2}\right), 2.35\left(\mathrm{~s}, 3 \mathrm{H}, \mathrm{COCH}_{3}\right), 7.32-$ $7.80(\mathrm{~m}, 5 \mathrm{H}, \mathrm{ArH}), 7.42(\mathrm{~s}, 1 \mathrm{H}, \mathrm{NH}) .{ }^{13} \mathrm{CNMR}(100 \mathrm{MHz}, \delta$, ppm, DMSO) 107.8-172.6 Ar-C), 134.6 (C=N), 14.4, 27.3 $\left(\mathrm{CH}_{3}\right), 61.4\left(\mathrm{CH}_{2}\right), 165.4,195.4(\mathrm{CO}) .{ }^{13} \mathrm{C} \mathrm{NMR}(100 \mathrm{MHz}$, $\delta$, ppm, DMSO) 108.8-156.6 (Ar-C), 14.2, 21.8, $26.9\left(\mathrm{CH}_{3}\right)$, $61.4\left(\mathrm{CH}_{2}\right), 165.4,195.4$ (CO). LC-MS: m/z $336\left(\mathrm{M}^{+}\right)$. Anal. Calcd for $\mathrm{C}_{16} \mathrm{H}_{17} \mathrm{~N}_{3} \mathrm{O}_{3} \mathrm{~S}$ (331): C, 57.99; H, 5.17; N, 12.68; S, 9.68. Found: C, 57.9; H, 5.1; N, 12.6; S, 9.6.

General Procedure for Synthesis of 1-(4-((1H-Benzotriazol-1-yl)methylamino)benzoyl)-3-methyl-4-(2-(substituted phenylthiazol-2-yl) hydrazono)-1H-pyrazol5(4H)-ones $7 \mathbf{a}-\mathbf{d}$

To a various ethyl 3-oxo-2-(2-(substituted phenylthiazol-2-yl) hydrazono) butanoate $\mathbf{6 a}-\mathbf{d}$ (0.002 mole) dissolved in glacial acetic acid $(20 \mathrm{ml})$, a solution of $4-((1 \mathrm{H}-$ benzotriazol-1-yl) methyl amino) benzo hydrazide 4 (0.002 mole) in $25 \mathrm{ml}$ of glacial acetic acid was added and the mixture was refluxed 10-12 h. It was then cooled and allowed to stand overnight. The resultant solid was filtered off, dried and crystallized from methanol to give desired product1-(4-((1H-benzotriazol-1-yl)methyl amino) benzoyl)3-methyl-4-(2-(substituted phenylthiazol-2-yl) hydrazono)1H-pyrazol-5(4H)-ones 7a-d.

\section{Physical Properties and Spectral Data}

1-(4-((1H-benzo[d][1,2,3]triazol-1-yl)methylamino) benzoyl)-3-methyl-4-(2-(4-phenyl thiazol-2-yl) hydrazono)-1H-pyrazol-5(4H)-one 7a

Yield 72\%; m.p. 206-208 ${ }^{\circ} \mathrm{C}$. IR (v, $\left.\mathrm{cm}^{-1}, \mathrm{KBr}\right) 3034-$ 3086 (C-Haromatic), 2920, $1465\left(\mathrm{CH}_{3}, \mathrm{CH}_{2}\right), 1290(\mathrm{C}-\mathrm{N})$, 1725-1765 $(\mathrm{C}=\mathrm{O}), 1695-1540(\mathrm{C}=\mathrm{N}), 3369(\mathrm{~N}-\mathrm{H}), 1597$, 1504 (Thiazole ring), $1550(\mathrm{C}-\mathrm{S}-\mathrm{C}) .{ }^{1} \mathrm{HNMR}(400 \mathrm{MHz}, \delta$, ppm, DMSO-d $\left.\mathrm{d}_{6}\right) 2.35\left(\mathrm{~s}, 3 \mathrm{H}, \mathrm{CH}_{3}\right), 5.64\left(\mathrm{~s}, 2 \mathrm{H}, \mathrm{CH}_{2}\right)$, 6.80-7.82 (m, 14H, ArH), 11.62, 6.97 (s, 2H, NH). ${ }^{13} \mathrm{C}$ NMR (100 MHz, $\delta$, ppm, DMSO) 110.8-151.6 (Ar-C), 11.6 $\left(\mathrm{CH}_{3}\right), 59.8\left(\mathrm{CH}_{2}\right), 164.3,170.5(\mathrm{CO}), 172.1-129.3(\mathrm{C}=\mathrm{N})$. LC-MS: $m / z 543\left(\mathrm{M}^{+}\right)$. Anal. Calcd for $\mathrm{C}_{27} \mathrm{H}_{21} \mathrm{~N}_{9} \mathrm{O}_{2} \mathrm{~S}(535)$ : C, 60.55; H, 3.95; N, 23.54; S, 5.99. Found: C, 60.5; H, $3.9 ; \mathrm{N}, 23.5 ; \mathrm{S}, 5.9$.

1-(4-((1H-benzo[d] $[1,2,3]$ triazol-1-yl) methylamino) benzoyl)-4-(2-(4-(4-bromophenyl)thiazol-2-yl) hydrazono)3-methyl-1H-pyrazol-5(4H)-one $7 \mathrm{~b}$

Yield 68\%; m.p. 206-208 ${ }^{\circ} \mathrm{C}$. IR $\left(v, \mathrm{~cm}^{-1}, \mathrm{KBr}\right) 3034-$ 3086 (C-H aromatic), 2920, $1465\left(\mathrm{CH}_{3}, \mathrm{CH}_{2}\right), 1290(\mathrm{C}-\mathrm{N})$, 1725-1765 $(\mathrm{C}=\mathrm{O}), 1695-1540(\mathrm{C}=\mathrm{N}), 3369(\mathrm{~N}-\mathrm{H}), 1597$, 1504 (Thiazole ring), $1550(\mathrm{C}-\mathrm{S}-\mathrm{C}), 550(\mathrm{C}-\mathrm{Br}) .{ }^{1} \mathrm{H}$ NMR (400 MHz, $\delta$, ppm, DMSO-d $\left.)_{6}\right) 2.35$ (s, 3H, $\left.\mathrm{CH}_{3}\right), 5.64$ (s,
2H, $\left.\mathrm{CH}_{2}\right), 6.80-7.82(\mathrm{~m}, 14 \mathrm{H}, \mathrm{ArH}), 11.62,6.97$ (s, 2H, $\mathrm{NH}) .{ }^{13} \mathrm{C}$ NMR (100 MHz, $\delta$, ppm, DMSO) 110.8-151.6 $(\mathrm{Ar}-\mathrm{C}), 11.6\left(\mathrm{CH}_{3}\right), 59.8\left(\mathrm{CH}_{2}\right), 164.3,170.5(\mathrm{CO}), 172.1-$ $129.3(\mathrm{C}=\mathrm{N}), 123.8(\mathrm{C}-\mathrm{Br})$. LC-MS: $m / z 628\left(\mathrm{M}^{+}\right)$. Anal. Calcd for $\mathrm{C}_{27} \mathrm{H}_{20} \mathrm{~N}_{9} \mathrm{O}_{2} \mathrm{SBr}$ (613): C, 52.77; H, 3.28; Br, 13.00; N, 20.52; S, 5.22. Found: C, 52.7; H, 3.2; Br, 12.9; N, 20.5; S, 5.1 .

1-(4-((1H-benzo[d][1,2,3] triazol-1-yl) methylamino) benzoyl)-4-(2-(4-(4-methoxyphenyl)thiazol-2-yl) hydrazono)-3-methyl-1H-pyrazol-5(4H)-one 7c

Yield 64\%; m.p. $213-216^{\circ} \mathrm{C}$. IR (v, $\left.\mathrm{cm}^{-1}, \mathrm{KBr}\right) 3034-$ 3086 (C-H aromatic), 2920, $1465\left(\mathrm{CH}_{3}, \mathrm{CH}_{2}\right), 1290(\mathrm{C}-$ $\mathrm{N})$, 1725-1765 $(\mathrm{C}=\mathrm{O}), 1695-1540(\mathrm{C}=\mathrm{N}), 3369(\mathrm{~N}-\mathrm{H})$, 1597, 1504 (Thiazole ring), $1550(\mathrm{C}-\mathrm{S}-\mathrm{C}), 1148(\mathrm{C}-\mathrm{O})$, 1205 (R-O-Ar). ${ }^{1} \mathrm{H}$ NMR (400 MHz, $\delta$, ppm, DMSO-d 6 ) $3.65\left(\mathrm{~s}, 3 \mathrm{H}, \mathrm{OCH}_{3}\right), 2.35\left(\mathrm{~s}, 3 \mathrm{H}, \mathrm{CH}_{3}\right), 5.64\left(\mathrm{~s}, 2 \mathrm{H}, \mathrm{CH}_{2}\right)$, 6.80-7.82 (m, 13H, ArH), 11.62, 6.97 (s, 2H, NH). ${ }^{13} \mathrm{C}$ NMR (100 MHz, $\delta, \mathrm{ppm}, \mathrm{DMSO})$ 110.8-151.6 (Ar-C), $\left.11.6\left(\mathrm{CH}_{3}\right), 56.2\left(\mathrm{O}-\mathrm{CH}_{3}\right), 59.8\left(\mathrm{CH}_{2}\right), 164.3,170.5 \mathrm{CO}\right)$, 172.1-129.3 (C=N). LC-MS: m/z $580\left(\mathrm{M}^{+}\right)$. Anal. Calcd for $\mathrm{C}_{28} \mathrm{H}_{23} \mathrm{~N}_{9} \mathrm{O}_{3} \mathrm{~S}$ (565): C, 59.46; H, 4.10; N, 22.29; S, 5.67. Found: C, 59.4; H, 4.0; N, 22.2; S, 5.6.

1-(4-((1H-benzo[d] [1,2,3] triazol-1-yl) methylamino)benzoyl)-3-methyl-4-(2-(4-p-tolyl thiazol-2-yl) hydrazono)1H-pyrazol-5(4H)-one 7d

Yield 67\%; m.p. $211-213{ }^{\circ} \mathrm{C}$. IR $\left(v, \mathrm{~cm}^{-1}, \mathrm{KBr}\right) 3034-$ 3086 (C-H aromatic), $1290(\mathrm{C}-\mathrm{N}), 2920,1465\left(\mathrm{CH}_{3}, \mathrm{CH}_{2}\right)$, 1725-1765 $(\mathrm{C}=\mathrm{O}), 1695-1540(\mathrm{C}=\mathrm{N}), 3369(\mathrm{~N}-\mathrm{H}), 1597$, 1504 (Thiazole ring), 1550 (C-S-C). ${ }^{1} \mathrm{H}$ NMR (400 MHz, $\delta$, ppm, DMSO-d 6 ) $2.42\left(\mathrm{~s}, 3 \mathrm{H}, \mathrm{CH}_{3}\right), 2.35\left(\mathrm{~s}, 3 \mathrm{H}, \mathrm{CH}_{3}\right)$, $5.64\left(\mathrm{~s}, 2 \mathrm{H}, \mathrm{CH}_{2}\right), 6.80-7.82(\mathrm{~m}, 13 \mathrm{H}, \mathrm{ArH}), 11.62,6.97$ (s, 2H, NH). ${ }^{13} \mathrm{C}$ NMR (100 MHz, $\delta$, ppm, DMSO) $110.8-$ 151.6 (Ar-C), 11.6, 21.7 $\left(\mathrm{CH}_{3}\right), 59.8\left(\mathrm{CH}_{2}\right), 164.3,170.5$ (CO), 172.1-129.3 (C=N). LC-MS: m/z $564\left(\mathrm{M}^{+}\right)$. Anal. Calcd for $\mathrm{C}_{28} \mathrm{H}_{23} \mathrm{~N}_{9} \mathrm{O}_{2} \mathrm{~S}(549)$ : C, 61.19; H, 4.22; N, 22.94; S, 5.83. Found: C, 61.1; H, 4.2; N, 22.9; S, 5.8.

\section{Biological Screening}

\section{Antibacterial activities}

Antibacterial activities of all the compounds were studied against Gram-positive Bacteria (Bacilus subtilis and Staphyllococcus aureus) and Gram-negative Bacteria (E. coil and Klebsiella promioe) at a concentration of $50 \mu \mathrm{g} / \mathrm{ml}$ by Agar cup plate method. Methanol system was used as control in this method. Under similar condition using sulphonamide as a standard for comparison carried out control experiment. The area of inhibition of zone measured in 
Table 1. Antibacterial activity of the compounds $7 \mathbf{a}-\mathbf{d}$

\begin{tabular}{|c|c|c|c|c|}
\hline \multirow{3}{*}{ Compounds } & \multicolumn{4}{|c|}{ Zone of Inhibition(mm) (Activity Index) ${ }^{\text {std }}$} \\
\hline & \multicolumn{2}{|c|}{ Gram + ve } & \multicolumn{2}{|c|}{ Gram - ve } \\
\hline & Bacillus subtilis & Staphylococcus aureus & Kllebsiella promioe & E. coil \\
\hline $7 \mathbf{a}$ & $82(0.92)$ & $50(0.84)$ & $78(0.90)$ & 71(0.93) \\
\hline $7 b$ & $88(0.98)$ & $56(0.94)$ & $84(0.97)$ & $73(0.96)$ \\
\hline $7 c$ & $86(0.96)$ & $58(0.98)$ & $82(0.95)$ & $74(0.97)$ \\
\hline $7 d$ & $85(0.95)$ & $54(0.91)$ & $80(0.93)$ & $70(0.92)$ \\
\hline Sulphonamide & 89 & 59 & 86 & 76 \\
\hline
\end{tabular}

(Activity Index) $^{\text {std }}=$ Zone of Inhibition of the sample/ Zone of Inhibition of the standard.

$\mathrm{mm}$. Compound $\mathbf{7 b}$ and $\mathbf{7 c}$ found more active against the above microbes. Other compounds were found more active against the above microbes. The antibacterial activities all compounds are shown in Table 1.

\section{Antifungal activity}

The fungicidal activity of all the compounds was studied at $1000 \mathrm{ppm}$ concentration in vitro. Plant pathogenic organisms listed in Table 2. The antifungal activities of all the samples were measured by cup plate method. ${ }^{36}$ Each of the plant pathogenic strains on potato dextrose agar (PDA) medium. Such a PDA medium contained potato 200 gms, dextrose 20 gms, agar 20 gms and water 1 liter. Five days old cultures were employed. The compounds to be tested were suspended (1000 ppm) in a PDA medium and autoclaved at $120^{\circ} \mathrm{C}$ for $15 \mathrm{~min}$, at $15 \mathrm{~atm}$ pressure. These medium were poured into sterile Petri plate and the organisms were inoculated after cooling the Petri plate. The percentage inhabitation for fungi was calculated after five days using the formula given below.

\section{Percentage of inhibition $=100(X-Y) / X$}

Where, X: Area of colony in control plate

Y: Area of colony in test plate

The fungicidal activity all compounds are shown in Table 2.

The antifungal activity of all the compounds measured for various plant pathogens. Inspection of the result shown in Table 2 indicates that all compounds are good toxic for fungi. These compounds almost inhibit the fungi about

Table 2. Antifungal activity of the compounds $7 \mathbf{a}-\mathbf{d}$

\begin{tabular}{ccccc}
\hline $\begin{array}{c}\text { Comp. } \\
\text { No. }\end{array}$ & $\begin{array}{c}\text { Penicillium } \\
\text { expansum }\end{array}$ & $\begin{array}{c}\text { Botrydepladia } \\
\text { thiobromine }\end{array}$ & $\begin{array}{c}\text { Nigrosspora } \\
\text { sp. }\end{array}$ & $\begin{array}{c}\text { Rhizopus } \\
\text { nigricuns }\end{array}$ \\
\hline $\mathbf{7 a}$ & 78 & 69 & 78 & 54 \\
$7 \mathbf{b}$ & 89 & 88 & 86 & 72 \\
$\mathbf{7 c}$ & 87 & 86 & 85 & 75 \\
$\mathbf{7 d}$ & 76 & 75 & 73 & 74 \\
\hline
\end{tabular}

$89 \%$. Hence produced compounds can be employed as garden fungicides. Further work in the direction is in progress.

\section{RESULTS AND DISCUSSION}

The compound 4 (hydrazide) has been synthesized successfully as the Mannich reaction reported previously. ${ }^{23-27}$ The synthesis of $5 \mathrm{a}-\mathrm{d}$ has been performed based on the method reported. ${ }^{34,35}$ From these compounds the novel compounds $6 \mathrm{a}-\mathrm{d}$ have been synthesized. The compounds $6 \mathrm{a}-\mathrm{d}$ reacted with 4 to give the corresponding compounds $7 \mathrm{a}-\mathrm{d}$. All the compounds were confirmed on the basis of the elemental analysis and spectroscopic investigation. IR spectrum of 4 revealed characteristic bands at 3450, 1630 $\left(\mathrm{NH}_{2}\right)$ and confirmatory by ${ }^{1} \mathrm{H} \mathrm{NMR} \delta 3.95\left(\mathrm{~s}, 2 \mathrm{H}, \mathrm{NH}_{2}\right)$. Further, IR spectroscopic investigation of $6 \mathrm{a}-\mathrm{d}$ reveals bands at $1580(\mathrm{C}=\mathrm{N})$ and ${ }^{1} \mathrm{H}$ NMR $\delta 7.42(\mathrm{~s}, 1 \mathrm{H}, \mathrm{NH})$.

IR spectra of compounds $7 \mathbf{a}-\mathbf{d}$ shows $1290(\mathrm{C}-\mathrm{N})$, $3369(\mathrm{~N}-\mathrm{H}), 1695-1540(\mathrm{C}=\mathrm{N})$ and ${ }^{1} \mathrm{H}$ NMR 11.62, 6.97 $(\mathrm{s}, 2 \mathrm{H}, \mathrm{NH})$. The examination of these data reveals that the IR band and ${ }^{1} \mathrm{H}$ NMR signals are appropriate to the correspond structure of compound.

The final structure of all compounds was confirmed by ${ }^{13} \mathrm{C}$ NMR and LC-MS data, i.e. The compounds 7 a shows the molecular ion peak $\mathrm{m} / \mathrm{z} 543$ give the molecular weight of $7 \mathbf{a}$ i.e. 535 . All these facts confirm the structures $7 \mathbf{a}-\mathbf{d}$.

\section{CONCLUSION}

A number of aryl azo pyrazole derivatives have been synthesized and characterized by elemental as well as spectral analysis. All these compounds screened for antibacterial and antifungal activity. The compounds $\mathbf{7 b}$ and $7 \mathbf{c}$ exhibited more antibacterial activity comparable to the standard drug Sulphonamide. The antifungal activity of all the compounds measured for various plant pathogens. Inspection of the result indicates that all compounds are good toxic for fungi. Hence produced compounds can be 
employed as garden fungicides. Further work in the direction is in progress.

Acknowledgments. The publication cost of this paper was supported by the Korean Chemical Society.

\section{REFERENCES}

1. Elguero, J.; Goya, P.; Jagerovic, N.; Silva, A. M. S., In Targets in Heterocyclic Systems; Attanasi, O. A., Spi nelli, D., Eds, Italian Society of Chemistry: Roma, Italy, 2002; Vol. 6, p 52.

2. Bekhit, A. A.; Ashour, H. M.; Guemei, A. A. Arch. Pharm. (Weinheim) 2005, 338, 167.

3. Pattan, S. R.; Rabara, P. A.; Pattan, J. S.; Bukitagar, A. A.; Wakale, V. S.; Musmade, D. S. Indian J. Chem. 2009, 48, 1453

4. Patel, B. P.; Patel, H. S.; Shah, P. J. Bulgarian Chemical Communications 2010, 42, 274.

5. El-Hawash, S. A.; Habib, N. S.; Kassem, M. Arch. Pharm. Chem. Life Sci. 2006, 339, 564.

6. Hiremith, S. P.; Rudresh, K.; Saundan, A. R. Indian J. Chem. 2002, 41B(2), 394.

7. Salih, N. A. Turk J. Chem. 2008, 32, 229.

8. Metwally, K. A.; Abdel-Aziz, L. M.; Lashine, el-S. M.; Husseiny, M. I.; Badawy, R. H. Bioorg. Med. Chem. 2006, 14(24), 8675.

9. Souza, F. R.; Souza, V. T.; Ratzlaff, V.; Borges, L. P.; Bonacorso, H. G.; Olivera, M. R. Eur. J. Pharma. 2002, 45(2), 141.

10. McTavish, J. R. Pain and Profits: The History of the Headache and Its Remediesin America; New Brunswick, NJ: Rutgers University Press: 2004; p 25.

11. Palaska, E.; Aytemir, M.; Uzbay, I. T.; Erol, D. Eur. J. Med. Chem. 2001, 36, 539.

12. Rajendra, P. Y.; Lakshmana, R. A.; Prasoona, L.; Murali, K.; Ravi, K. P. Bioorg. Med. Chem. Lett. 2005, 15, 5030.

13. Ozdemir, Z.; Kandilici, H. B.; Gumusel, B.; Calis, U.; Bilgin, A. A. Eur. J. Med. Chem. 2007, 42, 373.

14. Ruhogluo, O.; Ozdemir, Z.; Calis, U.; Gumusel, B.; Bilgin, A. A. Arzneimitte Forschung 2005, 55, 431.
15. Rostom, S. A. Bioorg. Med. Chem. 2006, 14(19), 6475.

16. Abdallah, M. A.; Riyadh, S. M.; Abbas, I. M.; Gomha, S. M. J. Chinese Chemical Soc. 2005, 52, 987.

17. Sabaa, M. W.; Oraby, E. H.; Abdul Naby, A. S.; Mohamed, R. R. Polym. Degrad. Stab. 2006, 91, 911.

18. Sabaa, M. W.; Oraby, E. H.; Abdul Naby, A. S.; Mohamed, R. R. J. Appl. Polym. Sci. 2006, 101, 1544.

19. Miyara, H. Bull. Chem. Soc. Jpn. 1963, 36, 382.

20. Khalifa, H.; Issa, M. Egypt J. Chem. 1974, 17, 581.

21. Azarifar, D.; Shaabanzadeh, M. Molecules 2002, 7, 885.

22. Amir, M.; Siddiqui, A. A.; Rizwan, S. Oriental J. Chem. 2003, 19(3), 629.

23. Bhatt, A. K.; Shah, P. R.; Karadiyya, H. G.; Patel, H. D., Oriental J. Chem. 2003, 19(3), 643.

24. Patel, K. V.; Singh, A. E-Journal of Chem. 2009, 6(1), 281.

25. Kartritzky, A. R.; Rachwal, S.; Rachwal, B. J. Chem. Soc. Perkin Trans. 1987, 1, 805.

26. Kartritzky, A. R.; Manju, K.; Singh, S. K.; Meher, N. K. Tetrahedron 2005, 61, 2555.

27. Katritzky, A. R.; Lan, X.; Yang, J. Z.; Denisko, O. V. Chem. Rev. 1998, 98, 409.

28. Sanna, P.; Carta, A.; Nikookar, M. E. Eur. J. Med. Chem. 2000, 35, 535 .

29. Kopanska, K.; Najda, A.; Zebrowska, J.; Chomicz, L.; Piekarczyk, J.; Myjak, P.; Bretner, M. Bioorg. Med. Chem. 2004, 12, 2617.

30. Boido, A.; Vazzana, I.; Mattioli, F.; Sparatore, F. Il Farmaco 2003, 58, 33.

31. Biagi, B.; Calderone, V.; Giorgi, I.; Scartoni, V.; Livi, O.; Baragatti, B.; Martinotti, E. Il Farmaco 2001, 56, 841.

32. Mishra, S.; Srivastava, S. K.; Srivastava, S. D. Indian. J. Chem. 1997, 36B, 826.

33. Vogel, A. I. Vogel's Text Book of Practical Organic Chemistry, 5th ed.; 1989; pp 896 and 1163.

34. Dodson, R. M.; King, C. L. J. Am. Chem. Soc. 1945, 67, 2242.

35. Carroll King, L.; Hlavek, R. J., J. Am. Chem. Soc. 1950, 72, 3722 .

36. Barry, A. L. The Antimicrobial Susceptibility Test: Principal and Practices, 4th ed.; Lea \& Febiger: Philadelphia, 1976; p 180. 